\title{
Global Behavior of the Fourth - Order Difference Equation
}

\section{Mehmet Emre Erdogan}

Abstract - In this article, the global asymptotic stability of the fourth order differential equation

$$
x_{n+1}=\frac{\alpha x_{n} x_{n-2}}{x_{n-1}\left(\beta+\gamma x_{n-3}\right)}
$$

where $\alpha, \beta$ and $\gamma$ are positive constants and non-negative initial conditions is examined.

Index Terms - Difference Equation, Asymptotic Stability, Global Behavior,

2010 Mathematics Subject Classification: 35B40, 39A10;

\section{INTRODUCTION}

The disciplines that examine subjects such as population, finance, probability, genetics need difference equations in mathematical models that have been created to explain real-life situations. Therefore, the necessity of some techniques that can be used in the investigation of the resulting equations reveals the importance of difference equations. Therefore, interest towards difference equations has increased.

Even if difference equations appear in a simple form, it is actually quite difficult to fully understand the behavior of their solutions, see [1-10] and the references cited therein. Until now, many studies have been done on the stability of nonlinear difference equations. For example:

Yang et al. [1] investigated the global asymptotic stability of the difference equation

$$
x_{n+1}=\frac{x_{n-1} x_{n-2}+x_{n-3}+a}{x_{n-1}+x_{n-2} x_{n-3}+a} .
$$

Kulenovic, Ladas and Sizer et al. [2] studied the behavior of rational recursive sequence

$$
x_{n+1}=\frac{\alpha x_{n}+\beta x_{n-1}}{\gamma x_{n}+\delta x_{n-1}} .
$$

Elabbasy and colleagues et al. [3] investigated and study some special cases of the difference equation

$$
x_{n+1}=\frac{\alpha x_{n-l} x_{n-k}}{b x_{n-p}-c x_{n-q}} .
$$

Abdul Khaliq and Elsayed et al. [4] studied behavior and obtained some special cases of the difference equation

$$
x_{n+1}=\frac{\alpha x_{n} x_{n-l}}{\beta x_{n-m}+\gamma x_{n-l}} .
$$

See also [5-14]. Our aim is examine the global behavior of the following fourth-order rational difference equation

$$
x_{n+1}=\frac{\alpha x_{n} x_{n-2}}{x_{n-1}\left(\beta+\gamma x_{n-3}\right)}
$$

where $\alpha, \beta$ and $\gamma$ are positive constants and non-negative initial conditions that will serve as the basis for such modeling.

Definition 1.1. [15] Let $I$ be some interval of real numbers and let

$$
f: I^{4} \rightarrow I
$$

be a continuously differentiable function.

Then for every set of initial conditions $x_{0}, x_{-1}, x_{-2}, x_{-3} \in I$, the difference equation

$$
x_{n+1}=f\left(x_{n}, x_{n-1}, x_{n-2}, x_{n-3}\right), n=0,1,2, \cdots
$$

has a unique solution $\left\{x_{n}\right\}_{n=-1}^{\infty}$.

A point $\bar{x} \in I$ is called an equilibrium point of Eq.(1.2) if $\bar{x}=f(\bar{x}, \bar{x}, \bar{x}, \bar{x})$

that is,

$$
x_{n}=\bar{x} \text { for } n \geq 0
$$

is a solution of Eq.(1.2), or equivalently, $\bar{x}$ is fixed point of $f$.

Definition 1.2. [15] Let $\bar{x}$ be an equilibrium point of Eq.(1.2)

(i) The equilibrium $\bar{x}$ of Eq.(1.2) is called locally stable if for every $\mathrm{o}>0$, there exists $\delta>0$ such that for all $x_{0}, x_{-1}, x_{-2}, x_{-3} \in I \quad$ with $\left|x_{0}-\bar{x}\right|+\left|x_{-1}-\bar{x}\right|+\left|x_{-2}-\bar{x}\right|+\left|x_{-3}-\bar{x}\right|<\delta$, we have

$$
\left|x_{n}-\bar{x}\right|<\text { ò for all } n \geq-3 \text {. }
$$

(ii) The equilibrium $\bar{x}$ of Eq.(1.2) is called locally asymptotically stable if it is locally stable, and if there exists $\gamma>0$ such that for all $x_{0}, x_{-1}, x_{-2}, x_{-3} \in I \quad$ with $\left|x_{0}-\bar{x}\right|+\left|x_{-1}-\bar{x}\right|+\left|x_{-2}-\bar{x}\right|+\left|x_{-3}-\bar{x}\right|<\gamma$, we have

$$
\lim _{n \rightarrow \infty} x_{n}=\bar{x}
$$

(iii) The equilibrium $\bar{x}$ of Eq.(1.2) is called global attractor if for every $x_{0}, x_{-1}, x_{-2}, x_{-3} \in I$ we have

$$
\lim _{n \rightarrow \infty} x_{n}=\bar{x}
$$


(iv) The equilibrium $\bar{x}$ of Eq.(1.2) is called global asymptotically stable if it is locally stable and a global attractor.

(v) The equilibrium $\bar{x}$ of Eq.(1.2) is called unstable if it is not stable.

(vi) The equilibrium $\bar{x}$ of Eq.(1.2) is called source or a repeller, if there exists $r>0$ such that for all $x_{0}, x_{-1}, x_{-2}, x_{-3} \in I \quad$ with $0<\left|x_{0}-\bar{x}\right|+\left|x_{-1}-\bar{x}\right|+\left|x_{-2}-\bar{x}\right|+\left|x_{-3}-\bar{x}\right|<r$, there exists $N \geq 1$ such that

$$
\left|x_{N}-\bar{x}\right| \geq r \text {. }
$$

The linearized equation of (1.2) about the equilibrium point $\bar{x}$ is

$$
y_{n+1}=p_{1} y_{n}+p_{2} y_{n-1}+p_{3} y_{n-2}+p_{4} y_{n-3}, n=0,1,2, \cdots
$$

where

$$
\begin{aligned}
& p_{1}=\frac{\partial f}{\partial x_{n}}(\bar{x}, \bar{x}, \bar{x}, \bar{x}) \\
& p_{2}=\frac{\partial f}{\partial x_{n-1}}(\bar{x}, \bar{x}, \bar{x}, \bar{x}) \\
& p_{3}=\frac{\partial f}{\partial x_{n-2}}(\bar{x}, \bar{x}, \bar{x}, \bar{x}) \\
& p_{4}=\frac{\partial f}{\partial x_{n-3}}(\bar{x}, \bar{x}, \bar{x}, \bar{x})
\end{aligned}
$$

The characteristic equation of (1.3) is

$$
\lambda^{4}-p_{1} \lambda^{3}-p_{2} \lambda^{2}-p_{3} \lambda-p_{4}=0
$$

Theorem 1.1. (The Linearized Stability Theorem)

(i) If all roots of (1.4) have absolute values less than one, then the equilibrium point $\bar{x}$ of (1.2) is locally asymptotically stable.

(ii) If at least one of the roots of (1.4) has absolute value greater than one, then the equilibrium point $\bar{x}$ of (1.2) is unstable.

(iii) The equilibrium point $\bar{x}$ of (1.2) is called saddle point if (1.4) has roots both inside and outside the unit disk.

Theorem 1.2. [15] Assume that $p_{i} \in \square, i=1,2, \cdots$. Then

$$
\sum_{i=1}^{4}\left|p_{i}\right|<1
$$

is a sufficient condition for the asymptotic stability of (1.4).

Theorem 1.3. [15] Let [ $p, q]$ be an interval of real numbers and assume that $f:[p, q]^{4} \rightarrow[p, q]$ is a continuous function satisfying the following properties:

(a) $f(x, y, z, t)$ is non-decreasing in $x, z \in[p, q]$ for each $y, t \in[p, q]$, and non-increasing in $y, t \in[p, q]$ for each $x, z \in[p, q]$;

(b) If $(m, M) \in[p, q] \times[p, q]$ is a solution of the system

$$
\begin{aligned}
& M=f(M, m, M, m) \text { and } m=f(m, M, m, M) \\
& \text { so } m=M .
\end{aligned}
$$

Then Eq.(1.2) has a unique equilibrium $\bar{x} \in[p, q]$ and every solution of Eq.(1.2) converges to $\bar{x}$.

\section{DYNAMICS OF}

\section{EQ.ERROR! REFERENCE SOURCE NOT FOUND.}

In this section, we investigate the dynamics of Error! Reference source not found. under the assumptions that all parameters in the equation are positive and the initial conditions are non-negative.

The Eq.Error! Reference source not found. has the equilibrium points obtained from the equation

$$
\bar{x}=\frac{\alpha \bar{x}^{2}}{\bar{x}(\beta+\gamma \bar{x})},
$$

so,

$$
\bar{x}^{2}(\gamma \bar{x}+\beta-\alpha)=0,
$$

then the equilibrium points are $\bar{x}_{1}=0$ and $\bar{x}_{2}=\frac{\alpha-\beta}{\gamma}$.

Theorem 2.1. The statements given below are true.

(i) If $3 \alpha<\beta$, then the equilibrium point $\bar{x}_{1}=0$ of Eq.Error! Reference source not found. is locally asymptotically stable.

(ii) If $3 \alpha>\beta$, then the equilibrium point $\bar{x}_{1}=0$ of

Eq.Error! Reference source not found. is a saddle point.

(iii) The equilibrium point $\bar{x}_{2}=\frac{\alpha-\beta}{\gamma}$ of

Eq.Error! Reference source not found. is unstable.

Proof. Let $f:(0, \infty)^{4} \rightarrow(0, \infty)$ be a function defined by

$$
f(u, v, w, t)=\frac{\alpha u w}{v(\beta+\gamma t)}
$$

So,

$$
\begin{aligned}
& \frac{\partial f}{\partial u}(\bar{x}, \bar{x}, \bar{x}, \bar{x})=\frac{\alpha}{\beta+\gamma \bar{x}}, \\
& \frac{\partial f}{\partial v}(\bar{x}, \bar{x}, \bar{x}, \bar{x})=-\frac{\alpha}{\beta+\gamma \bar{x}}, \\
& \frac{\partial f}{\partial w}(\bar{x}, \bar{x}, \bar{x}, \bar{x})=\frac{\alpha}{\beta+\gamma \bar{x}}, \\
& \frac{\partial f}{\partial t}(\bar{x}, \bar{x}, \bar{x}, \bar{x})=-\frac{\alpha \gamma \bar{x}}{(\beta+\gamma \bar{x})^{2}} .
\end{aligned}
$$

The linearized equation of Eq.Error! Reference source not found. about the equilibrium point $\bar{x}_{1}=0$ is

$$
x_{n+1}-\frac{\alpha}{\beta} x_{n}+\frac{\alpha}{\beta} x_{n-1}-\frac{\alpha}{\beta} x_{n-2}=0
$$


so, the characteristic equation of Eq.Error! Reference source not found. about the equilibrium point $\bar{x}_{1}=0$ is

$$
\lambda^{4}-\frac{\alpha}{\beta} \lambda^{3}+\frac{\alpha}{\beta} \lambda^{2}-\frac{\alpha}{\beta} \lambda=0
$$

From

Theorem

1.2

Eq.Error! Reference source not found. about the equilibrium point $\bar{x}_{1}=0$ is locally asymptotically stable if

$$
\left|\frac{\alpha}{\beta}\right|+\left|\frac{\alpha}{\beta}\right|+\left|\frac{\alpha}{\beta}\right|<1 .
$$

Thus $3 \alpha<\beta$. The proof of (ii) follows Theorem 1.1.

For (iii), the linearized equation of Eq.Error! Reference source not found. about the equilibrium point $\bar{x}_{2}=\frac{\alpha-\beta}{\gamma}$ has the form

$$
x_{n+1}-x_{n}+x_{n-1}-x_{n-2}+\frac{\alpha-\beta}{\alpha} x_{n-3}=0
$$

so, the characteristic equation of Eq.Error! Reference source not found. about the equilibrium point $\bar{x}_{2}=\frac{\alpha-\beta}{\gamma}$ is

$$
\lambda^{4}-\lambda^{3}+\lambda^{2}-\lambda+\frac{\alpha-\beta}{\alpha}=0 .
$$

From Definition 1.2 and Theorem 1.2, it is clear that Eq.Error! Reference source not found. about the equilibrium point $\bar{x}_{2}=\frac{\alpha-\beta}{\gamma}$ is unstable.

Then the proof is complete.

Theorem 2.2. The equilibrium point $\bar{x}_{1}=0$ of Eq.Error! Reference source not found. is globally asymptotically stable.

Proof. Let $g:[p, q]^{4} \rightarrow[p, q]$ is a function defined as $g(x, y, z, t)=\frac{\alpha x z}{y(\beta+\gamma t)}$. It can easily be seen that the $g(x, y, z, t)$ function is increased with respect to $x, z$ and decreasing with respect to $y, t$. Let us assume that $(m, M)$ is the solution of the system

$$
M=g(M, m, M, m) \text { and } m=g(m, M, m, M) .
$$

So

$$
M=\frac{\alpha M^{2}}{m(\beta+\gamma m)}, \quad m=\frac{\alpha m^{2}}{M(\beta+\gamma M)}
$$

and

$$
\begin{aligned}
& M m \beta+\gamma M m^{2}=\alpha M^{2} \\
& m M \beta+\gamma m M^{2}=\alpha m^{2}
\end{aligned}
$$

If we solve linear equation system, we obtain

$$
(M-m)(\alpha(M+m)+\gamma m M)=0
$$

Since $(\alpha(M+m)+\gamma m M)>0, M=m$.

From Theorem 1.3, the equilibrium point $\bar{x}_{1}=0$ is a global attractor of Eq.Error! Reference source not found. and then the equilibrium point $\bar{x}_{1}=0$ of Eq.Error! Reference source not found. is globally asymptotically stable by Definition 1.2.

Then the proof is complete.

Theorem 2.3. Let $\left\{x_{n}\right\}_{n=-3}^{\infty}$ be a solution of Eq.Error! Reference source not found. is bounded if $\alpha \leq 1$.

Proof. From Eq.Error! Reference source not found.

$$
\begin{aligned}
x_{1} & =\frac{\alpha x_{0} x_{-2}}{x_{-1}\left(\beta+\gamma x_{-3}\right)}<\alpha x_{0} x_{-2} \\
x_{2} & =\frac{\alpha x_{1} x_{-1}}{x_{0}\left(\beta+\gamma x_{-2}\right)}=\frac{\alpha \frac{\alpha x_{0} x_{-2}}{x_{-1}\left(\beta+\gamma x_{-3}\right)} x_{-1}}{x_{0}\left(\beta+\gamma x_{-2}\right)}=\frac{\alpha^{2} x_{-2}}{\left(\beta+\gamma x_{-2}\right)\left(\beta+\gamma x_{-3}\right)} \\
& <\alpha^{2} x_{-2}
\end{aligned}
$$

$$
\begin{aligned}
& x_{3}=\frac{\alpha x_{2} x_{0}}{x_{1}\left(\beta+\gamma x_{-1}\right)}=\frac{\alpha \frac{\alpha^{2} x_{-2}}{\left(\beta+\gamma x_{-2}\right)\left(\beta+\gamma x_{-3}\right)} x_{0}}{\frac{\alpha x_{0} x_{-2}}{x_{-1}\left(\beta+\gamma x_{-3}\right)}\left(\beta+\gamma x_{-1}\right)} \\
& =\frac{\alpha^{2} x_{-1}}{\left(\beta+\gamma x_{-1}\right)\left(\beta+\gamma x_{-2}\right)}<\alpha^{2} x_{-1} \\
& x_{4}=\frac{\alpha x_{3} x_{1}}{x_{2}\left(\beta+\gamma x_{0}\right)}=\frac{\alpha \frac{\alpha^{2} x_{-1}}{\left(\beta+\gamma x_{-1}\right)\left(\beta+\gamma x_{-2}\right)} \frac{\alpha x_{0} x_{-2}}{x_{-1}\left(\beta+\gamma x_{-3}\right)}}{\frac{\alpha^{2} x_{-2}}{\left(\beta+\gamma x_{-2}\right)\left(\beta+\gamma x_{-3}\right)}\left(\beta+\gamma x_{0}\right)} \\
& =\frac{\alpha^{2} x_{0}}{\left(\beta+\gamma x_{0}\right)\left(\beta+\gamma x_{-1}\right)}<\alpha^{2} x_{0} \\
& x_{5}=\frac{\alpha x_{4} x_{2}}{x_{3}\left(\beta+\gamma x_{1}\right)}=\frac{\alpha \frac{\alpha^{2} x_{0}}{\left(\beta+\gamma x_{0}\right)\left(\beta+\gamma x_{-1}\right)} \frac{\alpha^{2} x_{-2}}{\left(\beta+\gamma x_{-2}\right)\left(\beta+\gamma x_{-3}\right)}}{\frac{\alpha^{2} x_{-1}}{\left(\beta+\gamma x_{-1}\right)\left(\beta+\gamma x_{-2}\right)}\left(\beta+\gamma x_{1}\right)} \\
& =\frac{\alpha^{2} \frac{\alpha x_{0} x_{-2}}{x_{-1}\left(\beta+\gamma x_{-3}\right)}}{\left(\beta+\gamma x_{1}\right)\left(\beta+\gamma x_{0}\right)}=\frac{\alpha^{2} x_{1}}{\left(\beta+\gamma x_{1}\right)\left(\beta+\gamma x_{0}\right)}<\alpha^{2} x_{1}
\end{aligned}
$$

By iteration we obtain

$$
\left.\begin{array}{rl}
x_{4 k+1} & >x_{4 k+5} \\
x_{4 k} & >x_{4 k+4} \\
x_{4 k-1} & >x_{4 k+3} \\
x_{4 k-2} & >x_{4 k+2}
\end{array}\right\} \text { for } k=0,1,2, \cdots \text { if } \alpha \leq 1 \text {. }
$$


Then the subsequences $\left\{x_{n}\right\}_{n=-3}^{\infty}$ are decreasing and they are bounded to $M=\left\{x_{-2}, x_{-1}, x_{0}, x_{1}\right\}$ from above. This completes the proof.

\section{REFERENCES}

[1] Xiaofan Yang, On the global asymptotic stability of the difference equation $x_{n}=\frac{x_{n-1} x_{n-2}+x_{n-3}+a}{x_{n-1}+x_{n-2} x_{n-3}+a}$, Applied Mathematics and Computation, vol. 171(2), pp.857-861, 2005.

[2] M.R.S. Kulenović, G. Ladas, W.S. Sizer, On the recursive sequence $x_{n+1}=\frac{\alpha x_{n}+\beta x_{n-1}}{\gamma x_{n}+\delta x_{n-1}}$. Mathematical Sciences Research Hot-Line, vol. 2(5), pp.1-16, 1998.

[3] E.M. Elabbasy, H.A. El-Metwally, E.M. Elsayed, Global behavior of the solutions of some difference equations. Advances in Difference Equations, vol. 2011(1), 28, 2011.

[4] A. Khaliq, E.M. Elsayed, Qualitative study of a higher order rational difference equation, Hacettepe Journal of Mathematics and Statistics, vol. 47(5), 1128-1143, 2018.

[5] Mehmet Emre Erdogan, Cengiz Cinar, Ibrahim Yalcinkaya, On the dynamics of the recursive sequence $x_{n+1}=\frac{x_{n-1}}{\beta+\gamma x_{n-2}^{2} x_{n-4}+\gamma x_{n-2} x_{n-4}^{2}}$, Computers \& Mathematics with Applications, vol. 61(3), pp.533-537, 2011.

[6] Mehmet Emre Erdogan, Cengiz Cinar, Ibrahim Yalcinkaya, On the dynamics of the recursive sequence $x_{n+1}=\frac{\alpha x_{n-1}}{\beta+\gamma \sum_{k=1}^{t} x_{n-2 k} \prod_{k=1}^{t} x_{n-2 k}}$, Mathematical and Computer Modelling, vol. 54(5), pp.1481-1485, 2011

[7] Mehmet Emre Erdogan, Cengiz Cinar, On the dynamics of the recursive sequence $x_{n+1}=\frac{\alpha x_{n-1}}{\beta+\gamma \sum_{k=1}^{t} x_{n-2 k}^{p} \prod_{k=1}^{t} x_{n-2 k}^{q}}$, Fasciculi Mathematici, vol. 50, pp.59-66, 2013

[8] R. Abo-Zeid M.A. Al-Shabi, Global Behavior of a third order difference equation, Tamkang Journal of Mathematics, vol. 43(3), pp.375-383, 2012.

[9] Ravi P. Agarwal, Difference Equations and Inequalities: Theory, Methods, and Applications, Chapman \& Hall/CRC Pure and Applied Mathematics, 2000

[10] George L. Karakostas, Convergence of a Difference Equation Via The Full Limiting Sequences Method, Differential Equations and Dynamical Systems, vol. 1(4), pp.289-294, 1993.

[11] F. Belhannache, N. Touafek, R. Abo-Zeid, Dynamics of a third-order rational difference equation. Bulletin Mathematique de La Societe Des Sciences Mathematiques de Roumanie, vol. 59(1), pp.13-22, 2016.

[12] A. E. Hamza, A. M. Ahmed, A. M. Youssef, On the recursive sequence $x_{n+1}=\frac{a+b x_{n}}{A+B x_{n-1}^{k}}$. Arab Journal of Mathematical Sciences, vol. 17(1), pp.31-44, 2011.

[13] A. E. Hamza, E. M. Elsayed, Stability problem of some nonlinear difference eqauations, International Journal of Mathematics and Mathematical Sciences, vol. 21(2), pp.331-340, 1998.

[14] A. E. Grove, G. Ladas, M. Predescu, M. Radin, On the Gloval Character of the Difference Equation $x_{n+1}=\frac{\alpha+\gamma x_{n-(2 k+1)}+\delta x_{n-2 l}}{A+x_{n-2 l}}$. Journal of Difference Equations and Applications, vol. 9(2), pp.171-199, 2003.

[15] M. R. S. Kulenovic, G. Ladas, Dynamics of second order rational difference equations: with open problems and conjectures, Chapman and Hall/CRC, 2001.

[16] E. Camouzis, G. Ladas, Dynamics of Third-Order Rational Difference Equations with Open Problems and Conjectures, Chapman and Hall/CRC, 2008. 